\title{
TWO LOW-COST HUMAN COMPUTER INTERFACES FOR PEOPLE WITH SEVERE DISABILITIES
}

\author{
Mu-Chun Su', Yang-Han LeI ${ }^{2}$, Cheng-Hul Wu', Shi-Yong Su', Yu-XIang ZhaO' \\ 'Department of Computer Science and Information Engineering, National Central University, \\ Taiwan \\ ${ }^{2}$ Department of Electrical Engineering, Tamkang University, Taiwan
}

\begin{abstract}
The object of this paper is to present a set of techniques integrated into two low-cost human computer interfaces. Although the interfaces have many potential applications, one main application is to help the disabled persons to attain or regain some degree of independent communications and control. The first interface is a voice-controlled mouse and the second one is an accelerometer-based mouse.
\end{abstract}

Biomed Eng Appl Basis Comm, 2004(December); 16: 344-349.

Keywords: assistive technology, rehabilitation, computer interface, keyboard, mouse.

\section{INTRODUCTION}

With the growing use of computers the quality of our lives and even the whole society are dramatically changed. Owing to the rapidly increasing ability of computers to process information, we are going to be presented with computer-based world. For able-bodies people, access to computers can be taken for granted because conventional computer interfaces (e.g. a keyboard and a mouse) are designed with the ablebodies in mind. Unfortunately, people with physical disabilities cannot enjoy the benefits provided by computers on equal term. Therefore, how to lower or еven tear down the barriers between computers and users with disabilities is a very demanding task. Users with disabilities should be able to equally access standard computers and applications.

A wide range of interfaces between the user with disabilities and the device are available [1-8].

Received: Apr 19, 2004; Accepted: Oct 20, 2004 Correspondence: Mu-Chun Su, Professor

Department of Computer Science and Information Engineering, National Central University, Taiwan E-mail: muchun@csie.ncu.edu.tw
Traditional interfaces fall into one of three categories: switches, joystick controllers, and pneumatic devices. Each has its own advantages and disadvantages. For example, sip-puff pneumatic interface and chin strapped or mouth held joystick only require users to position the mouth and head in order to issue commands. However, users sacrifice a valuable degree of freedom and often feel uncomfortable. Recently, advances in electronic technologies and high-speed microprocessors have led to a new generation of interfaces for the disabled. The interface may be a complex system that allows a user to control equipments with the aid of eye movements [9-12], eye winks [13], EEG signals [14], EOG signals [15], infrared signals $[16]$, etc. Some of these interfaces usually provide few available controlled movements and require substantial motor coordination. Furthemore, they tend to be highly specialized and are generally cost prohibitive.

Since speech is a natural communication means for human being, the voice-operation feature is the idea control method for the large number of disabled people who cannot use conventional computer interfaces. Currently, several consumer speech recognition products (e.g. IBM's Via Voice and L\&H Dragon NaturallySpeaking, etc) are available. They can allow 
users to get computer tasks (e.g. send e-mail, dictation, and surf the Web) completed quickly and efficiently. With respect to text entry, these packages may be to some extent appealing and effective. Yet full access to computers is still not completely achieved for users with disabilities since text entry does not mean everything on computers. To have full access to computers, the user needs not only be able to enter text but also access to some other applications software, the computer desktop, etc. These speech recognition products may have problems of distinguishing commands (e.g. move the cursor to some where else) from text and spelling from text. In addition, if the user needs to work in more than one language, most of these commercial products have not yet provided an appealing solution to this problem. Therefore, a kind of complementary method should be developed to help the disabled persons to have full access to computers.

In our previous work [17], a voice-controlled human computer interface was proposed. It has several user-friendly features; however, the price paid for these advantages is that the system needs a DSP-based board (D6106A speech recognition module) which costs about US\$135. In this paper, we proposed a much cheaper way to implement a voice-controlled mouse which costs about US\$ 35 .

For non-speaking persons, the voice-controlled mouse is useless; therefore, we propose an accelerometer-based mouse which allows the user to use head movements to access computers.

This paper is organized into 4 sections. In the following section, we first introduce how to implement a low-cost voice-controlled mouse. Then the accelerometer-based mouse is presented in Section 3. Finally, the conclusions are given in Section 4.

\section{THE LOW-COST VOICE- CONTROLLED MOUSE}

The block diagram of the voice-controlled mouse is shown in Fig 1. With the voice-controlled mouse, the user can fully manipulate the computer via voice commands.

\subsection{System Design}

The complete system consists of three main modules: a speech recognition module, a microcontroller module, and a mouse circuit module.

(1) The speech recognition module: A voicecontrolled mouse allows the user to issue voice commands to move the cursor and/or click the buttons. In the system, the mouse-controlled functions include: up, down, left, right, clicking, double-clicking, rightclicking, and dragging. Therefore, it requires 8 different utterances for operating a mouse. In addition, if we want to use a voice command to stop the cursor we will need the ninth utterance. Therefore, we need a speech recognition module which can recognize at least 9 different utterances. In this research, we adopted a HL7003-based speech recognition module which is commercially available [18]. This speech recognition module can recognize 12 words and it takes about 3 seconds to recognize a word.

(2) The microcontroller module: In this module, we adopted the Intel-89C51 microprocessor which is available in shops at an affordable price. In our system, the major assignments of the $89 \mathrm{C} 51$ are to fetch the recognition results from the HL7003-based speech recognition module, to output two periodic waves with 90 ( phase lag to the mouse module to move the cursor, and to send signals to the mouse module to perform the clicking functions.

(3) The mouse circuit module: We used an IC HT4563 to implement the mouse module which performs the 8 mouse-controlled functions. The mouse circuit module is connected to the computer via a PS2 port.

\subsection{Experimental Results}

Fig. 2 shows the prototype of the voice-controlled mouse. The cost of the working prototype is approximately US $\$ 35$. Five subjects were asked to evaluate the proposed voice-controlled mouse. Before testing the prototype they were asked to choose their own 9 different utterances so that the speech recognition module can store the 9 corresponding template sequences into the system. Then they used the voice-controlled mouse to key in two English words (it includes 12 letters and one space key) via an on-screen keyboard. On average, each person took 10 minutes

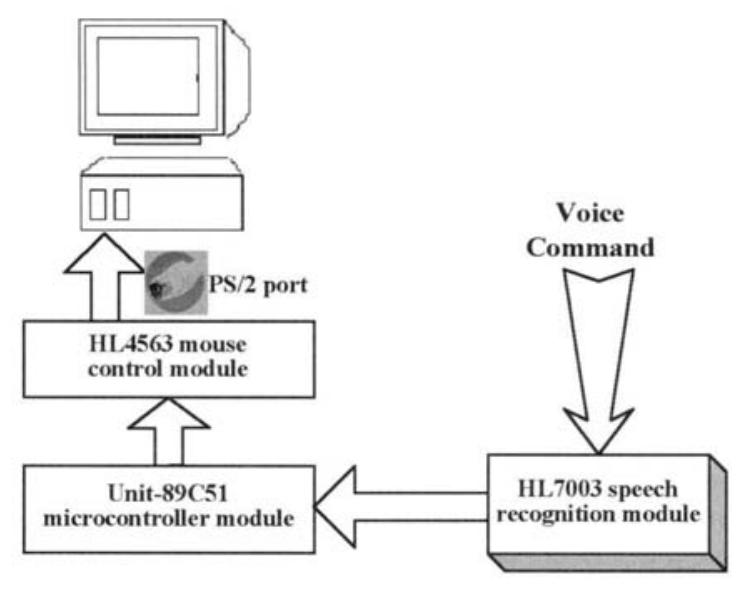

Fig. 1 The configuration of the low-cost voicecontrolled mouse. 
and 23 seconds. Since the speech recognition module takes 3 seconds to recognize a word and the cursor has to be moved across the on-screen keyboard backwards and forwards, it is not too surprised to find out the user needs a length of time to finish the typing task.

\section{THE ACCELEROMETER-BASED MOUSE}

The proposed accelerometer-based mouse allows the user to use head movements to access computers. The accelerometer-based mouse is fastened to a small platform and the platform is attached above a headset that is worn on the user's head. The user may tilt his or her head to move the cursor to any object on the window.

In fact, several different approaches to using head movements to access computers have been proposed [19-23]. Each has its own advantages and disadvantages. For example, the commercial products, the HeadMouse [19] and Head Master [20], are affordable for disabled persons in some developed countries; however, persons in some developing countries can not afford to use these commercial products if the price is not low enough. Considering the operation mode of the mouse, the user-friendly property is definitely an important factor which will affect a user to decide whether to use it. Intuitively, it will be very appealing if the speed of the cursor can vary with the inclination angle of the head. Most of the proposed approaches suffer from this kind of userfriendly property. Although the speed of the cursor can be in advance programmable via an application program the speed of the cursor is still fixed during the operation of the mouse [23].

In our system, the speed is proportional to the

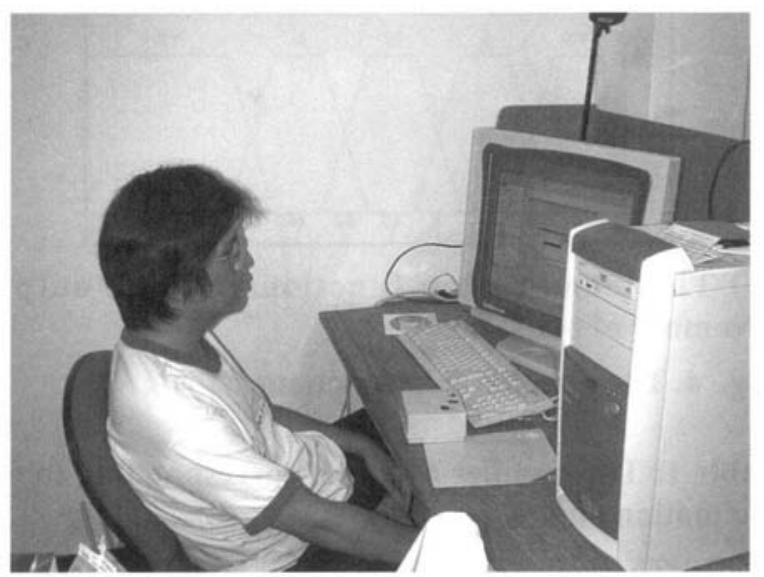

Fig. 2. The prototype of the voice-controlled mouse. inclination angle of the head. The larger the inclination angle is the faster the cursor is. Therefore, our proposed mouse can be operated in a more natural and intuitive way.

\subsection{System Design}

Fig. 3 shows the block diagram of the accelerometer-based mouse. The accelerometer-based mouse consists of the following five major units:

(1) The accelerometer module: A two-axial MEMS accelerometer is employed to measure the $\mathrm{X}$ and $\mathrm{Y}$-axis inclination angles of the movements. The MEMS accelerometer is capable of measuring "static" acceleration (e.g. earth's gravity) as well as "dynamic" acceleration (vibration). When an accelerometer is stationary (i.e. either lateral or vertical acceleration is present) the only force acting on the accelerometer is the gravitational force. The inclination angle is then the angle between the (vertical) gravitational force and the accelerometer sensing axis. The two-axial MEMS accelerometer is aligned in such a way that their $\mathrm{x}$-axes and $y$-axes pointed into the same direction, respectively. The two inclination angles can be computed as follows:

$$
\begin{gathered}
\alpha=\sin ^{-1}\left(A_{x} / g\right) \\
\beta=\sin ^{-1}\left(A_{y} / g\right)
\end{gathered}
$$

where $A x$ and $A y$ represent the two accelerometers' outputs, $\mathrm{g}$ is the acceleration due to gravity, and $\alpha, \beta$ are the $\mathrm{X}$ and $\mathrm{Y}$-axis inclination angles, respectively. In fact, when the operating region of the MEMS accelerometer is in the linear region the above two equations can be further approximated by the following two equations:

$$
\begin{gathered}
\alpha=k A_{x} \\
\beta=k A_{y}
\end{gathered}
$$

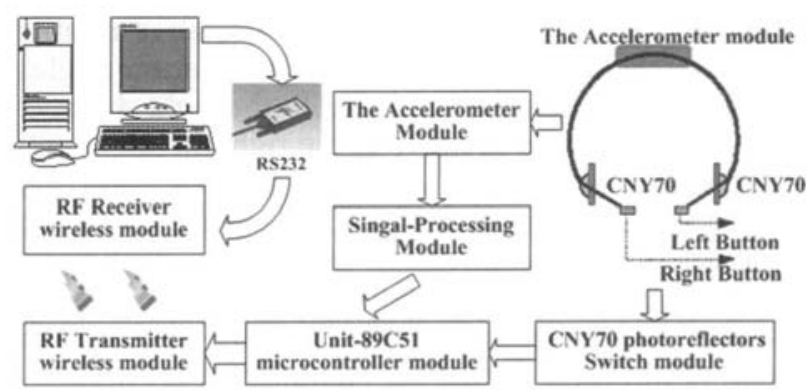

Fig. 3. The block diagram of the accelerometerbased mouse. 
where $\mathrm{k}$ is a constant.

(2) The signal processing module: The signal processing module consists of an offset amplifier and an 8-bit A/D convert. This module is employed to amplify the output of the accelerometer module and to digitize the amplified signals.

(3) The switch module: This module consists of a pair of CNY70 photoreflectors. The user can puff his or her cheek to trigger the photoreflectors to perform left clicking or right clicking of the mouse-controlled functions.

(4) The microcontroller module: In this module, we still adopted the Intel-89C51 microprocessor. The module receives the outputs of the accelerometer module and the switch module. Based on these outputs, the microprocessor implements all the mousecontrolled functions such as the clicking functions and the movements of the cursor. The outputs of the module can be connected to the serial port (i.e. RS232 port) of the computer either using a cable or using the wireless RF module.

(5) The wireless RF module: The module consisting of a transmitter and a receiver is used to transmit the outputs of the microcontroller module to the serial port of the computer. In this module, the ASK (amplitude-shift keying) scheme is adopted to transmit the signals.

\subsection{Mouse Operation}

The mouse can be operated in several ways. The simplest way is one degree of the inclination angle corresponds to one pixel movement of the cursor. Since the inclination angle is in the interval $\left[-90^{\circ}, 90^{\circ}\right]$ the displacement of the mouse is not able to meet the resolution of a commercially available monitor which usually is either $640 \times 480$ or $1280 \times 1024$. An alternative way is one degree of the inclination angle corresponds to $\mathrm{k}$ pixels movement of the cursor where $\mathrm{k}$ is an integer (e.g. $k=\lfloor 640 / 90\rfloor=7$ or $k=\lfloor 1280 / 90$ \rfloor$=14$ ). A crucial problem associated with this alternative way is that $\mathrm{k}$ pixels movement at a time may not allow a user to precisely point to an object. Therefore, another way is to use the relative rotation directions instead of the absolute inclination angles. For example, when the head tilts to the right direction the cursor continues to move to the right direction until a stop signal is triggered. This way is simple; however, the speed of the cursor is fixed. To change the speed, Chen adopted an application program to change the speed of the cursor [23]. Although the speed can be changed via an application program the speed of the cursor is still fixed during the operation of the mouse.

In this paper, we propose a natural and intuitive way to operate the mouse. The idea is as follows. The larger the inclination angle is the faster the speed of the cursor is. We first tried to quantize the interval of the inclination angle into six regions tabulated in Table I. When the inclination angle lies in one of the 6 regions the microcontroller module will continue to send signals to move the cursor with the corresponding number of pixels until the inclination angle restores back to the region $\left[0^{\circ}, 5^{\circ}\right]$.

Basically, the aforementioned approach allows the user to operate the mouse in a natural way. However, if the inclination angle is large the user usually needs to slightly tilt his or her head to the reverse direction when the cursor is going to arrive at the target object. Otherwise, the cursor will probably go beyond the target object. To overcome this side effect, we fuzzify the aforementioned crisp approach by using the following fuzzy rules:

If [Angle is Z] Then [Number of pixels is Z]
If [Angle is VS] Then [Number of pixels is VS]
If [Angle is S] Then [Number of pixels is S]
If [Angle is M] Then [Number of pixels is M]
If [Angle is L] Then [Number of pixels is L]
If [Angle is VL] Then [Number of pixels is VL]

The corresponding membership functions are shown in Fig. 4. We use the center average defuzzifier to compute the crisp output. From experiments, we find when we adopt the fuzzy approach the operation

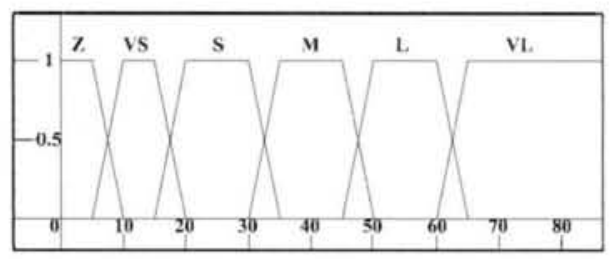

(a) The membership functions for the input "Angle".

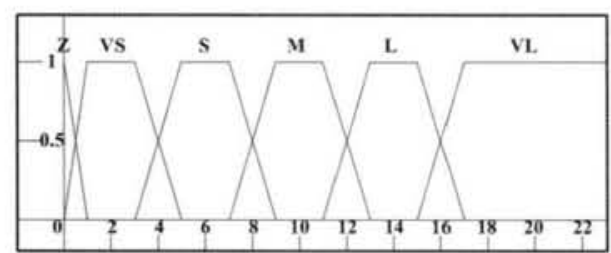

(b) The membership functions for the output "Number of pixels".

Fig. 4. The membership functions.

Table 1: The number of pixels corresponding to the inclination angles.

\begin{tabular}{|l|c|c|c|c|c|c|}
\hline angle & {$\left[0^{\circ}, 5^{\circ}\right]$} & {$\left[6^{\circ}, 15^{\circ}\right]$} & {$\left[16^{\circ}, 30^{\circ}\right]$} & {$\left[31^{\circ}, 45^{\circ}\right]$} & {$\left[46^{\circ}, 60^{\circ}\right]$} & {$\left[61^{\circ}, 90^{\circ}\right]$} \\
\hline pixels & 0 & 1 & 3 & 5 & 10 & 15 \\
\hline
\end{tabular}


of the mouse becomes more user friendly.

\section{EXPERIMENTAL RESULTS}

Fig. 5 shows the prototype of the accelerometerbased mousc. Two subjects were asked to operate the mouse. They were asked to key in nine Chinese characters (it includes 30 strokes) for five times. Table II tabulates the performance results. On average, the crisp approach and the fuzry approach required 285.6 seconds and 227.9 seconds, respectively. Obviously, the inclusion of fuzzy rules did improve the efficiency of the mouse.

\section{CONCLUSIONS}

The first aim of this project was to produce a lowcost voice-controlled mouse that allows a user to have full access to a computer via voice commands. The second aim is to design an accelerometer-based mouse which allows the user to use head movements to access

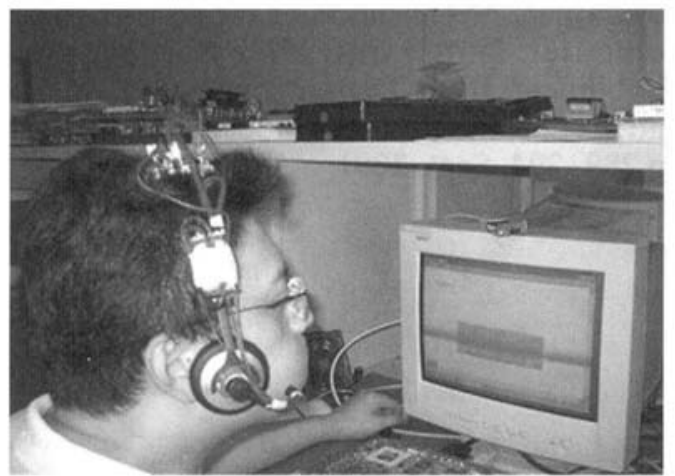

Fig. 5. The prototype of the accelerometer-based mouse.

Table 2: The performance of the accelerometerbased mouse.

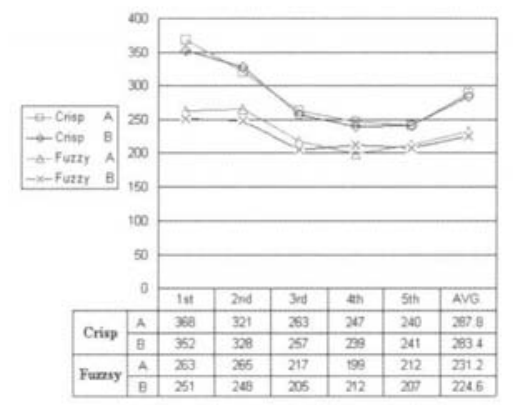

computers. In addition to the proposed human computer interfaces, we also implement an on-screen keyboard which is a graphical representation of a keyboard displayed on a computer screen. By using either one mouse, the user can enter text as if they were keyed in using a standard keyboard.

Simplicity, reliability, and low cost are of particular importance in many countries where only limited finance is available for aids for the disabled. The working prototypes of the mouse presented in this paper have achieved these main aims.

\section{ACKNOWLEDGEMENT}

This work is partially supported by the MOE Program for Promoting Academic Excellent of Universities under the grant number EX-91-E-FA06-44, the National Science Council, Taiwan, R.O.C, under the NSC 93-2524-S-008-002, and the Ministry of Economic Affairs under the 93-EC-17-A-02-SI-029.

\section{REFECRECES}

1. N. L. Lee and D. Keating, "Controllers for use by disabled people," computing \& control Engineering Journal, pp. 121-124, June, 1994.

2. C. Thomett, "Technical aids for the disabled," IEE Review, pp. 165-168, may 1989.

3. J. R. Gunderson, "Interfacing the motor-impaired for control and communication," Electronic Devices for Rehabilitation (Eds. J. G Webster, A. M. Cook, W. J. Tompkins, G. C. Vanderheiden), Johin Wiley \& Sons, NY, 1985.

4. A. W. S. Brown, "Inputs and how to activate a communication aid," Assistive Communication Aids (Ed P. M. Enderby), Churchill Livingstone, NY, 1987.

5. E. Peizer, E. J. Lorenze, and M. Dixon, "Environmental controls to promote independence in severely disabled elderly persons," Med. Instrum., vol. 16, no. 3, pp.171-173, May-June, 1982.

6. J. J. Vasa, "Electronic aids for the disabled and the elderly," Med. Instrum, vol. 16, no. 3, pp. 263-264, Sept-Oct, 1982.

7. W. J. Perkins and B. F. Stenning, "Control units for operation of computers by severely physical handicapped persons," J. Med. Eng. Technol., vol. 10, no. 1, pp. 21-23, Jan.-Feb., 1986.

8. M. J. Kilgallon, D. P. Roberts, and S. Miller, "Adapting personal computers for use by high-level quadripegics," Med. Instrum., vol. 21, no. 2, pp. 97. $102,1987$.

9. G. A. Rinard, R. W. Matteson, R. W. Quine, and R. 
S. Tegtmeyer, "An infrared system for determining ocular position," ISA Trans., vol. 19, no. 4, pp. 3-6, 1980.

10. J. R. Lacourse and F. C. Hladik, Jr., "An eye movement communication-control system for the disabled," IEEE Trans. Biomed. Eng., vol. 37, pp. 1215-1220, Dec. 1990.

11. K. Y. Tsai and J. H. Chang, "The development an eye-movement controlled man-machine interface for disable: optical eye-mouse," in Biomed. Eng. Soc. 1997 Annu. Symp., pp. 434-444, Taiwan, R.O.C., 1997.

12. G. Norris and E. Wilson, "The eye mouse, an eye communication device," IEEE 23rd Northeast Bioengineering Conference, pp. 66-67, May 1997.

13. R. Shaw, E. Crisman, A. Loomis, and Z. Laszewski, "The eye wink control interface: using the computer to provide the severely disabled with increased flexibility and comfort," The third Annual IEEE symposium on Computer-Based Medical Systems, pp. 105-111, 1990.

14. Z. A. Keirn and J. I. Aunon, "Man-machine communications through brain-wave processing," IEEE Eng. Med. Biol., pp. 55-57. Mar. 1990.

15. D. Kumar and E. Poole, "Classification of EOG for human computer interface," the Second Joint EMBS/BMES Conference, Vol. 1, pp. 23-26, Oct. 2002.
16. Y. -L. Chen, F. -T. Tang, W. H. Chang, M. -K. Wang, Y. -Y. Shih, and T. -S. Ku, "The new design of an infrared-controlled human-computer interface for the disabled," IEEE Trans. on Rehabilitation Engineering, vol. 7, no. 4, pp. 474-481, 1999.

17. M. C. Su and M. T. Chung, "A voice-controlled human-computer Interface for the disabled," IEE Computing \& Control Engineering Journal, vol. 12 , no. 5 , pp. 225-230, 2001.

18. HL7003 module. http://www.maolin.com.cn/cpjs.htm.

19. Head-Controlled Computer Access. http://www.orin.com/access/.

20. HeadMaster Plus. http://store.prentrom.com/.

21. Y. W. Kim and J. H. Cho, "A novel development of head-set type computer mouse using GYRO sensors for the handicapped," 2nd Annual International IEEE-EMB Conference on Microtechnologies in Medicine \& Biology, pp. 852-857, May 2002.

22. S. Lee, G. J. Nam, J. Chae, H. Kim, and A. J. Drake, "Two-dimensional position detection system with MEMS accelerometer for mouse application," Design Automation conference, pp.852 -857, June 2001.

23. Y. L. Chen, "Application of tilt sensors in humancomputer mouse interface for people with disabilities," IEEE Transactions on Neural Systems and Rehabilitation Engineering, vol. 9, no. 3, pp. $289-294$, Sept. 2001. 